\title{
On osculating spheres to rectifiable curves
}

\author{
by R. KrasnodęBSKI (Wrocław)
}

1. Introduction. In this paper we are concerned with $k$-dimensional spheres $S^{k}$ which osculate the rectifiable curve in an $n$-dimensional Euclidean space $E^{n}(k<n)$. There are two schemes in which the definition of the osculating sphere may be expressed. In one of them, say I, the definiens of the definition contains such a notion as order of contact. In the other, say II, the definiens contains information as to the way in which the osculating sphere is to be constructed. In the last case the definition may be called a constructive one.

The existence of the osculating sphere which has been defined according to scheme I does not depend on the regularity of the rectifiable curve: it is sufficient to assume that the curve differs sufficiently little from a spherical curve. A more precise formulation of this statement is contained in Theorem 1. We assume that the curve under consideration is rectifiable because this assumption enables us to use the notion of order of contact in the classical sense.

In considering differentiable curves the following problem arises: what is the order of contact between the osculating sphere and the curve at the inflexion points. This problem for $k=n-1$ has been investigated by Rachwal in [5], [6] and [7] under the assumption that the curve is of class $C_{n+1}$.

The most natural constructive definition of the osculating sphere is the following.

Let $\dot{P}_{v}, v=1,2, \ldots$, denote the set of $k+2$ different points $p_{v}^{1}, \ldots$ $\ldots, p_{v}^{k+2}$ on a curve $C$ and let $S_{v}^{k}$ be the sphere (possibly the $k$-dimensional hyperplane) through $p_{v}^{1}, \ldots, p_{v}^{k+2}$; let $\lim _{v \rightarrow \infty} P_{v}=p$ mean that for every $j=1, \ldots, k+2 \lim p_{v}^{j}=p$, where $p$ is a point on the curve. The symbol $P_{v}$ will stand also for the simplex (which may be degenerated) with vertices $p_{v}^{1}, \ldots, p_{v}^{k+2}$. The limit sphere, if it exists, of the sequence $\left\{S_{v}^{k}\right\}$ of spheres described on simplices $P_{v}$ such that $\lim _{v \rightarrow \infty} P_{v}=p$ will be denoted by $S_{\left\{P_{v}\right\}}^{k}$. 
Definition 1. If $S_{\left\{P_{v}\right\}}^{k}$ coincide for all sequences $\left\{P_{v}\right\}$ such that $\lim P_{v}=p$, then the common limit of all sequences $\left\{S_{v}^{k}\right\}$ is said to be $\rightarrow \infty$ the osculating sphere $S_{1}^{k}$ to $C$ at $p$.

In the case of $k=2, n=3$, Schwarz [8] has shown that if (i) the equation $r=r(t)$ of the curve is of class $C_{3}$, and (ii) the curvature and torsion are different from zero, then there exists an $S_{1}^{2}$ at $p$. The result depends on his theorem [8] in Advanced Calculus. By using Schwarz's theorem and applying his procedure one can obtain the length of the radius of the osculating sphere $S_{1}^{k}$ to the curve $C$ in $E^{n}(k<n)$ under the assumption that the radius vector $\boldsymbol{r}=\boldsymbol{r}(t)$ of $C$ is of class $C_{k+1}$ and that the curvatures $x_{1}, x_{2}, \ldots, x_{k}$ all exist and are different from zero.

Definition 1 may be modified by refusing, for example, the existence of the common limit of sequences of spheres $\left\{\boldsymbol{S}_{v}^{k}\right\}$ for all sequences $\left\{\boldsymbol{P}_{v}\right\}$ tending to $p$. Such a modification can be made in many ways and the respective osculating spheres exist under the respective assumption as to the regularity of the curve. Definition 4 and Theorem 2 give an answer to this question. We assume that the equation $r=r(s)$ of the curve for which the osculating sphere $S_{3}^{k}$ (i. e. the sphere according to Definition 4 ) is constructed at $s=0$ has at $s=0$ a derivative of $(k+1)$-th order in Denjoy's [1] sense. The proof of Theorem 2 is based on Lemma 1, which is a generalization of Schwarz's theorem. The Example in Section 5, which may be easily generalized both to arbitrary $k$ and to $n(k<n)$, illustrates the necessity of the omission of some sequences $\left\{P_{v}\right\}$ to ensure the existence of the osculating sphere $\mathbb{S}_{3}^{k}$ for curves satisfying the assumptions of Theorem 2 .

One can give another modifications of Definition 1 . Let $P_{v}^{\prime}, v=1$, $2, \ldots$, denote the set of $k$ different points $\left(p_{v}^{1}, \ldots, p_{v}^{k}\right)$ on the curve and let $L^{n-1}(p)$ be the $(n-1)$-dimensional hyperplane, if it exists, through $p$ which osculates the curve. $\operatorname{Lim} P_{v}^{\prime}=p$ is equivalent, as previously, to $\lim _{n \rightarrow \infty} p_{v}^{j}=p, j=1, \ldots, k$. The sphere (possibly a $k$-dimensional hyperplane) through $p_{v}^{1}, \ldots, p_{v}^{k}$ tangent to $L^{n-1}(p)$ at $p$ will be denoted by $S_{v}^{\prime k}$. The common limit sphere of the sequences $\left\{S_{v}^{\prime k}\right\}$ for all sequences $\left\{P_{v}^{\prime}\right\}$ tending to $p$, as $\nu \rightarrow \infty$, is said to be the osculating sphere $\mathcal{S}^{\prime k}$ to the curve at $p$. We could require the existence of the common limit sphere for some sequences $\left\{\boldsymbol{P}_{v}^{\prime}\right\}$.

Jarnik [4] gave eight definitions of the osculating circles to the plane curve. They are all expressed in the spirit of the above modifications of the classical definition. For each of his definitions he found necessary and sufficient conditions determining the regularity of the curve for which the osculating sphere exists. Fudali [3] generalized some of Jarnik's definitions for $k$ and $n$ arbitrary. He sought sufficient conditions for the 
curve for which an osculating sphere $\boldsymbol{S}^{k}$ would exist. In his definitions points $p_{v}^{1}, \ldots, p_{v}^{k+2}$ tend to $p$ maintaining a special order. The radius vector of the curve should have the $(k+1)$-th derivative at $p$ and satisfy some geometrical conditions which, together with the previous assumptions, are weaker than the fact that the radius vector under consideration is of class $C_{k+1}$.

2. Scheme I. Let the arc $s$ of the curve $C: r=r(s)$ belong to an open interval $U$ containing zero. Choose the origin in $E^{n}$ in such a way that it coincides with the point on $C$ for which $s=0$. Thus we have $r(0)=0$. Moreover, suppose that $r(s): U \rightarrow E^{n}$ is a 1-1 function. Points of $\boldsymbol{r}=\boldsymbol{r}(s)$ will be denoted by $p(s)$ except of $p(0)$, which we denote by $p$.

Definition 2. A $k$-dimensional sphere $S^{k}$ is said to be the osculating sphere $S_{2}^{k}$ at $p$ to the rectifiable curve $\boldsymbol{r}=\boldsymbol{r}(s)$ if: (i) $p$ lies on $S^{k}$ and (ii) $\lim \frac{d(s)}{s^{k+1}}=0$, where $d(s)$ is the distance from $p(s)$ to $S^{k}$.

By a $k$-spherical curve we mean a curve which lies entirely on a $k$-dimensional sphere.

THEOREM 1. The osculating sphere $S_{2}^{k}$ to the rectifiable curve $\boldsymbol{r}=\boldsymbol{r}(s)$ at $p$ exists if and only if

$$
\boldsymbol{r}(s)=\boldsymbol{r}^{*}(s)+\rho(s),
$$

where $\boldsymbol{r}=\boldsymbol{r}^{*}(s)$ is a rectifiable $k$-spherical curve and

$$
\lim _{s \rightarrow 0} \frac{\rho(s)}{s^{k+1}}=\mathbf{0} .
$$

Proof. Suppose that $r(s)$ may be expressed as in (1) and the curve $\boldsymbol{r}=\boldsymbol{r}^{*}(s)$ lies on a sphere $S^{k}$. The distance $d(s)$ of $p(s)$ to $S^{k}$ does not exceed $|p(s)|$. Hence it follows from (2) that the first part of the theorem is true, i. e. $S^{k}=S_{2}^{k}$.

Let us now assume that there is an osculating sphere $S_{2}^{k}$ to $\boldsymbol{r}=\boldsymbol{r}(s)$ at $p$. Project the curve perpendicularly on the hyperplane $E^{k+1}$ which contains $S_{2}^{k}$. We obtain a curve $\boldsymbol{r}=\boldsymbol{r}_{h}(s)$ and we have $\boldsymbol{r}(s)=\boldsymbol{r}_{h}(s)+\boldsymbol{\rho}_{1}(s)$. Then project the curve $r=r_{h}(s)$ on the sphere $S_{2}^{k}$ along its radii. We obtain a $k$-spherical curve $\boldsymbol{r}=\boldsymbol{r}^{*}(s)$, obviously rectifiable (both projections are analytical). Therefore we have $\boldsymbol{r}_{h}(s)=\boldsymbol{r}^{*}(s)+\boldsymbol{\rho}_{2}(s)$ and

$$
\boldsymbol{r}(s)=\boldsymbol{r}^{*}(s)+\boldsymbol{p}_{1}(s)+\boldsymbol{p}_{2}(s),
$$

where

$$
\left|\rho_{1}(s)+\rho_{2}(s)\right|=d(s)
$$

satisfies (ii) in Definition 2 by the existence of the osculating sphere $\boldsymbol{S}_{2}^{k}$. 
We now verify (3). Denote by $p_{h}(s)$ the projection of $p(s)$ on $E^{k+1}$; $p_{h}(s)$ may be defined as a point of contact between $E^{k+1}$ and a $(n-1)$-dimensional sphere $S^{n-1}$, with centre $p(s)$. We distinguish two cases:

$1^{\circ}$ Let $p_{h}(s)$ be different from the centre of $S_{2}^{k}$. If $p_{h}(s)$ lies on $S_{2}^{k}$, then we have $\rho_{2}(s)=0$ and obviously $\left|\rho_{1}(s)\right|=d(s)$. If $p_{h}(s)$ does not lie on $S_{2}^{k}$, let us enlarge the radius of $S^{n-1}$, without changing its centre, till it reaches a point of contact with $S_{2}^{k}$. This point, say $p^{*}(s)$, belongs to the curve $r^{*}(s)$ defined above. In fact, points $p_{h}(s)$ and $p^{*}(s)$ both lie on a radius of $S_{2}^{k}$. This is because $p_{h}(s)$ is the centre of all $k$-dimensional spheres which are sections of $E^{k+1}$ and $(n-1)$-dimensional spheres $S^{n-1}$.

From our construction we have: $p_{1}(s)=\overrightarrow{p_{h}(s) p(s)}, p_{2}(s)=\overrightarrow{p^{*}(s) p_{h}(s)}$, $\rho_{1}(s) \cdot p_{2}(s)=0$ and $\operatorname{dist}\left(p(s), p^{*}(s)\right)=d(s)$. Thus if $p_{h}(s)$ does not lie on $S_{2}^{k}(3)$ is also valid.

$2^{0}$ When, for some $s, p_{h}(s)$ and the centre of $S_{2}^{k}$ coincide, we take a sufficiently small interval $U^{\prime} \subset U$ containing zero such that for $s \in U^{\prime}$ dist $\left(p, p_{h}(s)\right)$ is smaller than the radius of $S_{2}^{k}$; then the construction from $1^{0}$ is admissible also in this case.

Consequently we define a vector function $\rho(s)$ as equal to $p_{1}(s)+p_{2}(s)$.

And this is the end of the proof.

\section{Preparatory lemmas.}

LEMMA 1. Let $f_{1}(t), \ldots, f_{k+1}(t)$ be continuous functions in an open interval $U$ containing zero and such that

(i) $\lim _{l \rightarrow 0} \frac{f_{h}(t)}{t^{l_{h}}}=a_{h}$, where $\left|a_{h}\right|<\infty, h=1, \ldots, k+1,1 \leqslant l_{1}<l_{2}<\ldots$ $\ldots<l_{k+1} ;$ moreover, let

(ii) $\left\{t_{v}^{1}, \ldots, t_{v}^{k+2}\right\}$ be such a sequence of $k+2$ values of $t$ in $U$ that for each $v=1,2, \ldots, t_{\nu}^{1}, \ldots, t_{\nu}^{k+2}$ are different;

(iii) $\lim _{v \rightarrow \infty} t_{v}^{j}=0$ for each $j=1,2, \ldots, k+2$;

(iv) there exist no subsequence $\{\bar{v}\}$ of $\{v\}$ and no pair $i_{0}, j_{0}\left(i_{0} \neq j_{0}\right)$ for which $\lim _{\bar{\nu} \rightarrow \infty} \frac{t_{\bar{v}}^{i_{0}}}{t_{\bar{v}}^{j_{0}}}=1$;

(v) at least for one index $h, a_{h}=0$;

then

$$
\lim _{v \rightarrow \infty} \frac{A_{v}}{V_{v}}=0
$$


where

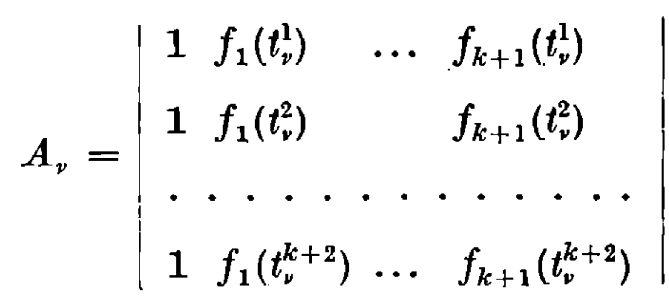

and

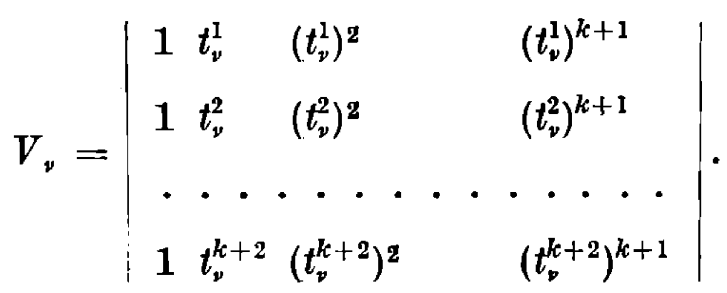

Proof. We consider two cases:

$1^{\circ} t_{v}^{j} \neq 0$ for any pair $j, v . A_{v}$ is the sum of the following components:

$$
A_{j_{1} \ldots j_{k+1}}=\varepsilon f_{1}\left(t_{\nu}^{j_{1}}\right) \ldots f_{k+1}\left(t_{\nu}^{j_{k+1}}\right), \quad \varepsilon= \pm 1,
$$

where $j_{1}, \ldots, j_{k+1}$ is an arbitrary permutation of the fundamental permutation $1,2, \ldots,(k+1)$. Vandermond's determinant $V_{v}$ is the product of $(k+1)(k+2) / 2$ factors:

$$
V_{v}=\varepsilon \prod_{1 \leqslant h<j \leqslant k+2}\left(t_{v}^{j}-t_{v}^{h}\right), \quad \varepsilon= \pm 1
$$

Having fixed a permutation $j_{1} \ldots j_{k+1}$ we order, for simplicity, the factors in (5) as follows. Set $t_{v}^{j_{1}}-t_{v}^{h^{\prime}}$ for some $h^{\prime}$ as the first factor; then take $\left(t_{v}^{j 2}-t_{v}^{h^{\prime \prime}}\right)\left(t_{v}^{j}-t_{v}^{h^{\prime \prime \prime}}\right)$ for some $h^{\prime \prime}, h^{\prime \prime \prime}$ as the second and third factors; then set three factors

$$
\left(t_{v}^{j_{3}}-t_{v}^{h^{\mathrm{IV}}}\right)\left(t_{v}^{j_{3}}-t_{v}^{h^{\mathrm{V}}}\right)\left(t_{v}^{j_{3}}-t_{v}^{h^{\mathrm{VI}}}\right)
$$

for some $h^{\mathrm{IV}}, h^{\nabla}, h^{\mathrm{\nabla I}}$ and so on. Such an arrangement of all the factors in (5) is obviously possible. In the quotient $A_{j_{1}} \cdots j_{k+1} / V_{\nu}$ we divide both the numerator and the denominator by

$$
t_{\nu}^{j_{1}}\left(t_{v}^{j_{2}}\right)^{2} \ldots\left(t_{v}^{j_{k+1}}\right)^{k+1}
$$

Then the denominator is a product of $(k+1)(k+2) / 2$ factors of the form $1-t_{v}^{j} / t_{\nu}^{h}$ and the numerator may be expressed as follows

$$
\varepsilon \frac{f_{1}\left(t_{v}^{j_{1}}\right)}{t_{v}^{j_{1}}} \cdot \frac{f_{2}\left(t_{v}^{j_{2}}\right)}{\left(t_{\nu}^{j_{2}}\right)^{2}} \cdot \ldots \cdot \frac{f_{k+1}\left(t_{v}^{j_{k+1}}\right)}{\left(t_{v}^{j_{k+1}}\right)^{k+1}}, \quad \varepsilon= \pm 1 .
$$

From (i) and ( $v$ ) we conclude that (6) converges to zero as $\nu \rightarrow \infty$ and from (iv) and (ii) we see that the absolute value of every factor $1-t_{v}^{j} / t_{v}^{h}$ 
in the denominator for every $v$ is greater than a positive number. Thus in this case (4) holds.

$2^{\circ} t_{v}^{j}=0$ for some $j$ and $v$. If there is a finite number of pairs $j, v$ for which $t_{v}^{j}=0$, then the reasoning in $1^{\circ}$ remains valid. If it is not the case, denote by $\left\{v^{\prime}\right\}$ the subsequence of $\{\nu\}$ for which $t_{v^{\prime}}^{j}=0$, where $j$ is tired and by $\left\{A_{v^{\prime}}^{j}\right\}$ and $\left\{\nabla_{v^{\prime}}^{j}\right\}$ the corresponding subsequences of $\left\{A_{v}\right\}$ and $\left\{\boldsymbol{V}_{*}\right\}$. In virtue of $f_{i}\left(t_{v^{\prime}}^{j}\right)=0, i=1, \ldots, k+1$, and since for any index $v^{\prime}$ there is one and only one upper index in $t_{v^{\prime}}^{j}$ for which $t_{v^{\prime}}^{j}$ equals to zero, we have

$$
A_{v^{\prime}}^{j}=\varepsilon\left|\begin{array}{ccc}
f_{1}\left(t_{v^{\prime}}^{j}\right) & & f_{k+1}\left(t_{v^{\prime}}^{1}\right) \\
\ldots & \ldots & \ldots \\
f_{1}\left(t_{v^{\prime}}^{j-1}\right) & \ldots & f_{k+1}\left(t_{v^{\prime}}^{j-1}\right) \\
f_{1}\left(t_{v^{\prime}}^{j+1}\right) & \ldots & f_{k+1}\left(t_{v^{\prime}}^{j+1}\right) \\
\ldots & \ldots & \ldots \\
f_{1}\left(t_{v^{\prime}}^{k+2}\right) & \ldots & f_{k+1}\left(t_{v^{\prime}}^{k+2}\right)
\end{array}\right|, \quad \varepsilon= \pm 1
$$

and

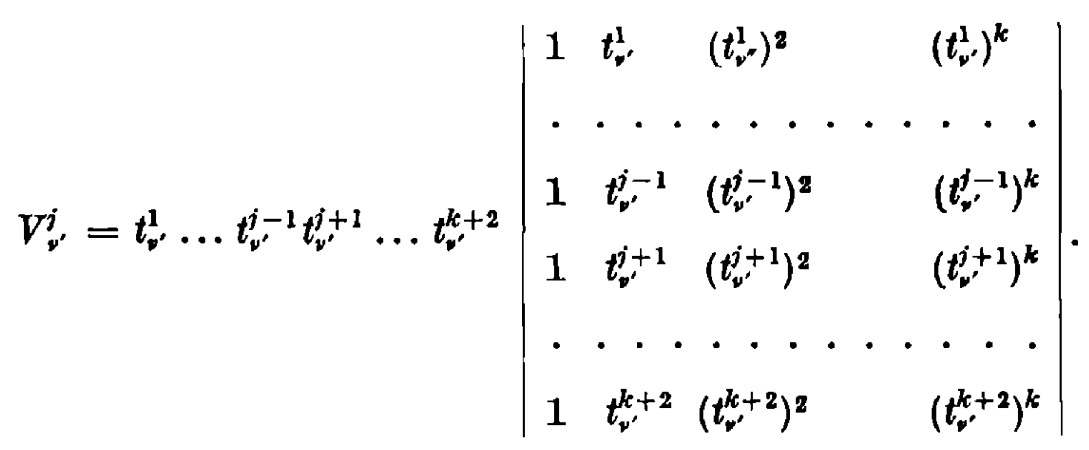

By similar arguments to those in $1^{\circ}$ one can easily prove that in this case $\lim A_{v^{\prime}}^{i} / V_{v^{\prime}}^{i}$ also equals zero. Thus, for all subsequences for which $\lim t_{v^{\prime}}^{i}=\stackrel{0}{\nu^{\prime} \rightarrow \infty}$, formula (4) is valid.

LEMma 2. Let $\left\{P_{v}\right\}$ and $\left\{P_{v}^{\prime}\right\}$ be two sequences of $m$-dimensional simplices in $E^{n}$; let $S^{m-1}$ and $S^{\prime m-1}$ be the spheres described on $P_{v}$ and $P_{v}^{\prime}$ respectively; let $R_{v}$ and $R_{v}^{\prime}$ be the radii of $S_{v}^{m-1}$ and $S_{v}^{\prime m-1}$, respectively. If

(i) the edges $d_{v, i j}$ of $P_{v}$ and $d_{v, i j}^{\prime}$ of $P_{v}^{\prime}(i, j=1, \ldots, m+1)$, joining two different vertices $A_{i}, A_{\gamma}$ and $A_{i}^{\prime}, A_{j}^{\prime}$ of $P_{v}$ and $P_{v}^{\prime}$, respectively, tend to zero as $v \rightarrow \infty$;

(ii) the edges of $P_{v}$ are related to the edges of $P_{v}^{\prime}$ in such a way that $\lim _{v \rightarrow \infty} d_{v, i j} / d_{v, i j}^{\prime}=1$;

(iii) there exists a lim?t $R$ of the sequence $\left\{R_{v}\right\}$;

then there exists a limit $R^{\prime}$ of the sequence $\left\{R_{v}^{\prime}\right\}$ and $R^{\prime}=R$. 
Proof. The radius $R_{y}$ of $S_{v}^{m-1}$ is a function of $m(m+1) / 2$ edges of $P_{v}$. By using Theorem $\Pi$ in Dimensional Analysis [2] we may express $R_{\mathrm{y}}$ as

$$
R_{v}=\varphi\left(\frac{d_{v, i j}}{d_{v, 12}}\right) d_{v, 12}
$$

where $\varphi$ is a positive continuous function of $m(m+1) / 2-1$ arguments $d_{v, 13} / d_{v, 12}, d_{v, 14} / d_{v, 12}, \ldots, d_{v, m m+1} / d_{v, 12}$. From (iii) we see that $\left\{R_{\nu}\right\}$ has a limit $R$ as $v \rightarrow \infty$ and $R$ is the radius of $S^{m-1}$.

As for $R_{v}$ we may write that

$$
R_{v}^{\prime}=\varphi\left(\frac{d_{v, i j}^{\prime}}{d_{v, 12}^{\prime}}\right) d_{v, 12}^{\prime}
$$

Now multiply every argument $d_{v, i j}^{\prime} / d_{v, 12}^{\prime}$ of $\varphi$ in (7) by $d_{v, 12} d_{v, i j} / d_{v, 12} d_{v, i j}$ and $d_{v, 12}^{\prime}$ by $d_{v, 12} / d_{v, 12}$. Then we obtain

$$
R_{v}^{\prime}=\varphi\left(\frac{d_{v, i j}^{\prime}}{d_{v, i j}} \frac{d_{v, 12}}{d_{v, 12}^{\prime}} \frac{d_{v, i j}}{d_{v, 12}}\right) \frac{d_{v, 12}^{\prime}}{d_{v, 12}} d_{v, 12}
$$

Thus it follows from (ii) and (iii) that there exists a limit of $\left\{R_{v}^{\prime}\right\}$ as $\nu \rightarrow \infty$ and that $\lim _{\nu \rightarrow \infty} R_{v}^{\prime}=\lim _{\nu \rightarrow \infty} R_{\nu}=R$.

4. Scheme II. The notations from the beginning of Section 2 remain valid. As in section 1 , denote by $\left\{P_{v}\right\}$ the sequence $p\left(s_{v}^{1}\right), \ldots, p\left(s_{v}^{k+2}\right)$ of $k+2$ different points of $\boldsymbol{r}(s)(\nu=1,2, \ldots)$. The same symbol $P_{v}$ will denote the simplex (which may be degenerated) with vertices $p\left(s_{v}^{1}\right), \ldots$ $\ldots, p\left(s_{v}^{k+2}\right)$. In what follows we only consider sequences $s_{v}^{j}, j=1, \ldots, k+2$, such that $\lim s_{v}^{i}=0$; thus $\lim p\left(s_{v}^{j}\right)=p$ also always holds.

Definition 3. The sequence $\left\{P_{\nu}\right\}$ is said to be normal if for any subsequence $\left\{P_{v^{\prime}}\right\}$ of $\left\{P_{v}\right\}$ and each pair $\left(i_{0}, j_{0}\right) \lim _{v^{\prime} \rightarrow \infty} s_{v^{\prime}}^{i_{0}} / s_{v^{\prime}}^{j_{0}} \neq 1$.

Definition 4. If $S_{\left\{P_{\nu}\right\}}^{k}$ coincide for all normal sequences $\left\{\boldsymbol{P}_{v}\right\}$, then the common limit sphere of all sequences of spheres $\left\{S_{v}^{k}\right\}$ is said to be the osculating sphere $S_{3}^{k}$ to $\boldsymbol{r}=\boldsymbol{r}(s)$ at $p$.

THEOREM 2. Suppose that the radius vector $\boldsymbol{r}(s)$, where $r$ is the arc of a rectifiable curve $C$ in $E^{n}$, can be expressed in a neighbourhood of $s=0$ in the form

$$
\boldsymbol{r}(s)=\boldsymbol{q}(s)+\boldsymbol{\rho}(s),
$$

where

$$
\boldsymbol{q}(s)=\boldsymbol{r}_{0}+\boldsymbol{r}_{1} s+\boldsymbol{r}_{2} s^{2}+\ldots+\boldsymbol{r}_{k+1} s^{k+1}
$$


$\boldsymbol{r}_{1}, \boldsymbol{r}_{2}, \ldots, \boldsymbol{r}_{k+1}$ are independent vectors and $\lim _{s \rightarrow 0} \frac{\rho(s)}{s^{k+1}}=0$. Then there exists at $p, i$ e. at the point for which $s=0$, an osculating sphere $S_{3}^{k}$ and it coincides with a k-dimensional osculating sphere $S^{\prime k}$ to the algebraic curve $\boldsymbol{r}=\boldsymbol{q}(s)$ at $p$.

Proof. $1^{0}$ Choose in $E^{n}$ an orthonormal coordinate system with basic vectors $e_{1}, e_{2}, \ldots, e_{n}$ and origin at $p$ and such that $r_{1}=a_{1}^{1} e_{1}$, $r_{2}=a_{2}^{1} e_{1}+a_{2}^{2} e_{2}, \ldots, r_{k+1}=a_{k+1}^{1} e_{1}+\ldots+a_{k+1}^{k+1} e_{k+1}$, where owing to the independence of $r_{1}, r_{2}, \ldots, r_{k+1}$ we have $a_{1}^{1} \cdot a_{2}^{2} \cdot \ldots \cdot a_{k+1}^{k+1} \neq 0$. Let $E^{k+1}$ denote the space generated by $e_{1}, \ldots, e_{k+1}$ (or by $r_{1}, \ldots, r_{k+1}$ ). The components $x^{1}(s), \ldots, x^{k+1}(s)$ of $\boldsymbol{r}(s)$ in the orthonormal coordinates are equal to

$$
x^{l}(s)=a_{l}^{l} s^{l}+\varepsilon_{l}(s), \quad l=1, \ldots, k+1,
$$

where $\varepsilon_{l}(s)=a_{l+1}^{l} s^{l+1}+\ldots+a_{k+1}^{l} s^{k+1}+\rho(s) e_{l}, l=1, \ldots, k+1$, so that

$$
\lim _{s \rightarrow 0} \frac{\varepsilon_{l}(s)}{s^{l}}=0, \quad l=1, \ldots, k+1
$$

$2^{0}$ For any normal sequence $\left\{P_{v}\right\}$ there is a neighbourhood $U_{1}$ of $p$ on $C$ such that all simplices $P_{v}$ whose vertices belong to $U_{1}$ are nondegenerate. Indeed, denote by $Q_{v}$ the perpendicular projection of $P_{v}$ on $E^{k+1}$. If $Q_{\nu}$ is not degenerate, neither is $P_{v}$. The volume of $Q_{\nu}$, vol $\left(Q_{v}\right)$, is equal to the determinant $c\left|1 x^{1}\left(s^{j}\right) \ldots x^{k+1}\left(s^{j}\right)\right|, j=1, \ldots, k+2$, where $c$ is a constant different from zero. Using (9) we obtain a determinant with "double" columns except the first one:

$\operatorname{vol}\left(Q_{v}\right)$

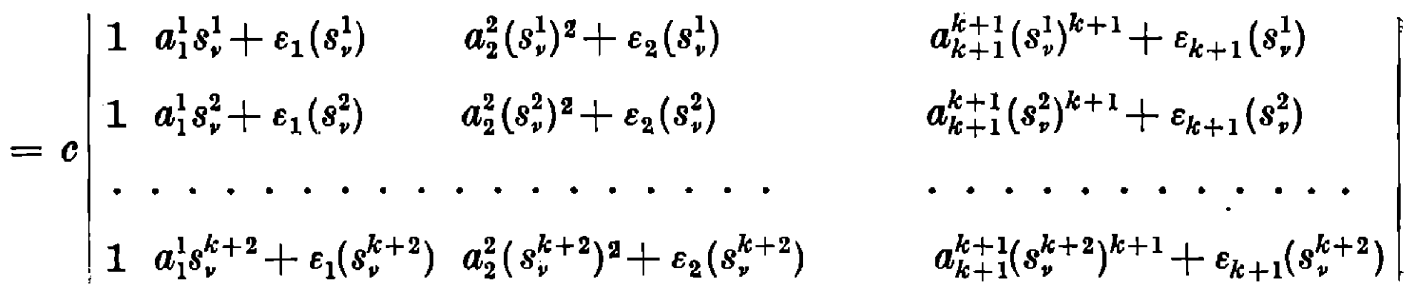

Thus $c^{-1} \operatorname{vol}\left(Q_{\nu}\right)$ is the sum of $2^{k+1}$ determinants with single columns: $c^{-1} \operatorname{vol}\left(Q_{v}\right)=a_{1}^{1} a_{2}^{2} \ldots a_{k+1}^{k+1} V_{v}+A_{2, \nu}+A_{3, v}+\ldots+A_{2^{k+1}, v}$, where $V$, is Vandermond's determinant of $s_{v}^{1}, s_{v}^{2}, \ldots, s_{v}^{k+2}$ and the remaining determinants satisfy the assumptions of Lemma 1. Assumptions (ii), (iii) and (iv) are obviously satisfied. For each $A_{h, v}, h=2,3, \ldots, 2^{k+1}$, the sequence $l_{1}$, $l_{2}, \ldots, l_{k+1}$ defined in (i) of Lemma 1 is the following: $1,2, \ldots, k+1$; thus assumption (i) of Lemma 1 is also satisfied. Further, each $A_{h, v}$, $h=2,3, \ldots, 2^{k+1}$, contains at least one column like 


$$
\left[\begin{array}{c}
\varepsilon_{l}\left(s_{v}^{1}\right) \\
\varepsilon_{l}\left(s_{v}^{2}\right) \\
\cdot \cdot \cdot \cdot \\
\varepsilon_{l}\left(s_{v}^{k+2}\right)
\end{array}\right] \quad(1=1,2, \ldots, k+1)
$$

which is the $(l+1)$-th column in $A_{h, v}$. Thus again because of $\left(l_{1}, l_{2}, \ldots, l_{k+1}\right)$ $=(1,2, \ldots, k+1)$ in each $A_{h, v}, h=2,3, \ldots, 2^{k+1}$, and in virtue of $(10)$ assumption (v) in Lemma 1 is fulfilled. Therefore, by use of Lemma 1, we have

$$
\lim _{\nu \rightarrow \infty} \frac{A_{h, \nu}}{V_{\nu}}=0, \quad h=2,3, \ldots, 2^{k+1}
$$

Since $V_{v} \neq 0$ for any $v$ there exists such an index $v_{0}$ that for $v>v_{0}$ $\operatorname{vol}\left(Q_{\nu}\right)>0$.

$3^{\circ}$ We have

$$
\begin{aligned}
& \lim _{v \rightarrow \infty} \frac{\rho\left(s_{v}^{i}\right)-\rho\left(s_{v}^{j}\right)}{\left|\boldsymbol{q}\left(s_{v}^{i}\right)-\boldsymbol{q}\left(s_{v}^{j}\right)\right|} \\
= & \lim _{v \rightarrow \infty} \frac{\rho\left(s_{v}^{i}\right)-\rho\left(s_{v}^{j}\right)}{s_{v}^{i}-s_{v}^{i}} \cdot \frac{s_{v}^{i}-s_{v}^{j}}{\left|\boldsymbol{r}_{1}\left(s_{v}^{i}-s_{v}^{j}\right)+\ldots+\boldsymbol{r}_{k+1}\left[\left(s_{v}^{i}\right)^{k+1}-\left(s_{v}^{j}\right)^{k+1}\right]\right|}, \quad i \neq j .
\end{aligned}
$$

Let us divide the numerator and the denominator of the second factor of the second term in (11) by $s^{i}-s^{j}$. Then. we see that this factor tends to $\left|\boldsymbol{r}_{1}\right|^{-1}$ as $v \rightarrow \infty$. From Lemma 1 in the special case where $A$, and $V_{v}$ are two order determinants we deduce that the first factor tends to zero as $\nu \rightarrow \infty$. Thus

$$
\lim _{v \rightarrow \infty} \frac{\rho\left(s_{v}^{i}\right)-\rho\left(s_{v}^{j}\right)}{\boldsymbol{q}\left(s_{v}^{i}\right)-\boldsymbol{q}\left(s_{v}^{j}\right)}=0
$$

Hence

$$
\lim _{\nu \rightarrow \infty}\left|\frac{\boldsymbol{r}\left(s_{v}^{i}\right)-\boldsymbol{r}\left(s_{v}^{j}\right)}{\boldsymbol{q}\left(s_{v}^{i}\right)-\boldsymbol{q}\left(s_{v}^{j}\right)}\right|=\lim _{\nu \rightarrow \infty}\left|\frac{\boldsymbol{q}\left(s_{v}^{i}\right)-\boldsymbol{q}\left(s_{v}^{j}\right)}{\left|\boldsymbol{q}\left(s^{i}\right)-\boldsymbol{q}\left(s^{j}\right)\right|}+\frac{\rho\left(s_{v}^{i}\right)-\boldsymbol{\rho}\left(s_{v}^{j}\right)}{\left.\mid \boldsymbol{q}\left(s^{i}\right)-\boldsymbol{q}\left(s^{j}\right)\right\}}\right|=1 .
$$

$4^{\circ}$ Denote by $C^{\prime}$ the curve $\boldsymbol{r}=\boldsymbol{q}(s)$ and $P_{v}^{\prime}$ the set of points $p^{\prime}\left(s_{v}^{i}\right), \ldots, p^{\prime}\left(s_{v}^{k+2}\right)$ of $C^{\prime}$. The directions of vectors $\overrightarrow{p\left(s_{v}^{i}\right) p\left(s_{v}^{j}\right)}$ and of vectors

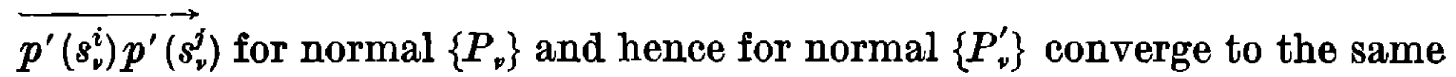
limit as $\nu \rightarrow \infty$. In fact, we have from (8), (12) and (13) 
(14) $\lim _{v \rightarrow \infty} \frac{\boldsymbol{q}\left(s_{v}^{i}\right)-\boldsymbol{q}\left(s_{v}^{j}\right)}{\left|\boldsymbol{q}\left(s_{v}^{i}\right)-\boldsymbol{q}\left(s_{v}^{j}\right)\right|}=\lim _{\nu \rightarrow \infty} \frac{\boldsymbol{r}\left(s_{v}^{i}\right)-\boldsymbol{r}\left(s_{v}^{j}\right)-\rho\left(s_{v}^{i}\right)+\rho\left(s_{v}^{j}\right)}{\left|\boldsymbol{q}\left(s_{v}^{i}\right)-\boldsymbol{q}\left(s_{v}^{j}\right)\right|}=\lim _{\nu \rightarrow \infty} \frac{\boldsymbol{r}\left(s_{v}^{i}\right)-\boldsymbol{r}\left(s_{v}^{j}\right)}{\left|\boldsymbol{q}\left(s_{v}^{i}\right)-\boldsymbol{q}\left(s_{v}^{j}\right)\right|}$

$$
\begin{aligned}
& =\lim _{v \rightarrow \infty} \frac{\boldsymbol{r}\left(s_{v}^{i}\right)-\boldsymbol{r}\left(s_{v}^{j}\right)}{\left|\boldsymbol{r}\left(s_{v}^{i}\right)-\boldsymbol{r}\left(s_{v}^{j}\right)\right|} \cdot \frac{\boldsymbol{r}\left(s_{v}^{i}\right)-\boldsymbol{r}\left(s_{v}^{j}\right)}{\left|\boldsymbol{q}\left(s_{v}^{i}\right)-\boldsymbol{q}\left(s_{v}^{j}\right)\right|} \\
& =\lim _{v \rightarrow \infty} \frac{\boldsymbol{r}\left(s_{v}^{i}\right)-\boldsymbol{r}\left(s_{v}^{j}\right)}{\left|\boldsymbol{r}\left(s_{v}^{i}\right)-\boldsymbol{r}\left(s_{v}^{j}\right)\right|} .
\end{aligned}
$$

$5^{o}$ Through the midpoints of the segments $p^{\prime}\left(s_{v}^{1}\right) p^{\prime}\left(s_{v}^{j}\right), j=2, \ldots, k+2$, perpendicularly to $p^{\prime}\left(s_{v}^{1}\right) p^{\prime}\left(s_{v}^{j}\right)$ draw $(n-1)$-dimensional hyperplanes $F_{v}^{\prime}(j)$. The point of intersection of these hyperplanes for $j=2, \ldots, k+2$ and a $(k+1)$-dimensional hyperplane $G_{v}^{\prime}$ containing $P_{v}^{\prime}$ is the centre of the sphere $S_{v}^{\prime k}$ described on $P_{v}^{\prime}$. The limit of $\left\{S_{v}^{\prime k}\right\}$ as $\nu \rightarrow \infty$ exists for all sequences $\left\{P_{v}^{\prime}\right\}$ and all these sequences have the same limit sphere $S^{k}$ with c'entre, say $o^{\prime}$.

Denote by $F_{\nu}(j), j=2, \ldots, k+2$, the $(n-1)$-dimensional hyperplanes drawn perpendicularly to $p\left(s_{v}^{1}\right) p\left(s_{v}^{j}\right)$ through the midpoints of $p\left(s_{v}^{1}\right) p\left(s_{v}^{j}\right)$ and by $G_{v}$ the $(k+1)$-dimensional hyperplanes containing $P_{v}$. If follows from (14) that for normal sequences $\left\{\boldsymbol{P}_{v}\right\}$

$$
\lim _{\nu \rightarrow \infty} F_{\nu}(j)=\lim _{\nu \rightarrow \infty} F_{\nu}^{\prime}(j), \quad j=2, \ldots, k+2,
$$

and

$$
\lim _{\nu \rightarrow \infty} G_{v}=\lim _{\nu \rightarrow \infty} G_{v}^{\prime} \text {. }
$$

The limit hyperplanes $F^{\prime}(j)$ and $E^{k+1}$ of the sequences $\left\{F_{v}^{\prime}(j)\right\}$ and $\left\{G_{v}^{\prime}\right\}$ respectively have two different common points $o^{\prime}$ and $p$. Obviously there exists one and only one $k$-dimensional hyperplane $E^{k} \subset E^{k+1}$ tangent to the curve $C^{\prime}$ at $p$. Thus any $k$ vectors among the $k+1$ vectors

$$
\lim _{v \rightarrow \infty} \frac{\boldsymbol{q}\left(s_{v}^{\mathrm{l}}\right)-\boldsymbol{q}\left(s_{v}^{j}\right)}{\mid \boldsymbol{q}\left(s_{v}^{1}\right)-\frac{\boldsymbol{q}}{\left(s_{v}^{j}\right) \mid}}, \quad j=2, \ldots, k+2,
$$

are independent. Therefore any $k$ hyperplanes among the $k+1$ hyperplanes $F^{\prime}(j), j=2, \ldots, k+2$, and $E^{k+1}$ intersect each other along the line passing through $p$ and $o^{\prime}$. It follows from (15) and (16) that the limit hyperplanes $F(j), j=2, \ldots, k+2$, of the sequences $\left\{F_{v}(j)\right\}$ for normal $\left\{P_{v}\right\}$ intersect each other in $E^{k+1}$ also along $p o^{\prime}$.

$6^{\circ}$ From Lemma 2 and (13). we conclude that there exists a limit $S_{3}^{k}$ for every sequence $\left\{\boldsymbol{S}_{v}^{k}\right\}$ of spheres described on $\boldsymbol{P}_{v}$ which are the members of the normal sequence $\left\{P_{v}\right\}$ and that the radii of $\mathcal{S}_{3}^{k}$ and $\mathcal{S}^{\prime k}$ have the same length. Thus it follows from the arguments in $5^{\circ}$ that the centres of $S_{3}^{k}$ and $S^{\prime k}$ coincide. 
Definitions 1,2 and 4 yield the same $k$-dimensional osculating sphere $S^{\prime k}$ to the algebraic curve $\boldsymbol{r}=q(s)$ at $p$. Thus from Theorem 2 we have the following

CoROLlary. The order of contact of $C$ and $S_{3}^{k}$ at $p$ is equal to $k+1$.

5. Example $(k=1, n=3)$. Consider a segment $C^{\prime}$ of the parabola

$$
q(s)=e_{1} s+e_{2} s^{2}
$$

for which $s \leqslant 5 / 8$. The are of $C^{\prime}$ denote by $\sigma$. Let $\sigma=0$ when $s=0$ and $\sigma>0$ when $s>0$. For different values of $\sigma, q_{\sigma}$ will stand for points of $C^{\prime}$. Consider two sequences of points on $C^{\prime}: q_{1 / 2}, q_{1 / 3}, \ldots$ and $q_{-1 / 2}$, $q_{-1 / 3}, \ldots$ Then construct isosceles triangles $\tau_{v}=q_{1 / v} b_{\nu} q_{1 /(v+1)}$ and $\tau_{-,}$ $=q_{-1 / v} b_{-\nu} q_{-1 /(v+1)}, v=2,3, \ldots$, such that

$$
q_{1 / v} b_{v}=q_{1 /(v+1)^{\prime}} b_{v}=\delta_{v}=\frac{1}{2}\left(\frac{1}{v}-\frac{1}{\nu+1}+\frac{1}{v^{3}}-\frac{1}{(\nu+1)^{3}}\right)
$$

and

$$
q_{-1 / v} b_{-\nu}=q_{-1 /(v+1)} b_{-\nu}=\delta_{\nu}
$$

(ii) any quadruple $b_{v}, b_{v+1}, b_{p+2}, b_{v+3}$ and any quadruple $b_{-v}, b_{-(p+1)}$, $b_{-(v+2)}, b_{-(v+3)}$, do not lie on the plane $E^{2}$ of $C^{\prime}$.

The polygon $q_{-1 / 2} b_{-2} q_{-1 / 3} b_{-3} \ldots q_{0} \ldots b_{3} q_{1 / 3} b_{2} q_{1 / 2}$ is the desired curve $C$ which satisfies the assumptions of Theorem 2 . The equation

$$
\boldsymbol{r}(s)=\boldsymbol{q}(s)+\rho(s)
$$

of $C$, where $s$ is now the are of $C$, determines the vector $p(s)$. We show that

$$
\lim _{s \rightarrow 0} \frac{\rho(s)}{s^{2}}=0 .
$$

Consider the case $s>0$ (for $s<0$ the proof is identical). The vector $\rho(s)$ is determined by the points: $p(s) \epsilon C$ and $p^{\prime}(s) \epsilon C^{\prime}$, i. e.

$$
\rho(s)=\overrightarrow{p^{\prime}(s) p(s)} .
$$

The length of the polygon $q_{0} \ldots b_{8} q_{1 / 3} b_{2} q_{1 / 2}$ is equal to $\sum_{p=2}^{\infty} 2 \delta_{v}=5 / 8$. Thus for $s=5 / 8$ we have $q_{1 / 2}=p(5 / 8)$. Therefore the points $p^{\prime}(5 / 8) \epsilon C^{\prime}$ and $q_{1 / 2} \in C$ yield the initial vector $\rho(5 / 8)$ of the vector function $p(s)$, which then slides with its origin on $C^{\prime}$ and its end on $C$ as $s$ decreases from $5 / 8$ to 0 .

Denote by $s_{v}$ the length of the are of $C$ (of the polygon) from $q_{0}$ to $q_{1 / p} ;$ it is easy to see that $s_{v}=\left(v^{2}+1\right) / \nu^{3}$ (Fig. 1). Two sequences $\{1 / v\}$ 
and $x_{v}$, where $x_{v}$ is the abscissa of $p\left(8_{v}\right)$ (or of $q_{1 / v}$ ), are equivalent. Hence

$$
\lim _{v \rightarrow \infty} \frac{e_{1} \rho\left(s_{v}\right)}{\left(s_{v}\right)^{2}}=\lim _{r \rightarrow \infty} \frac{s_{v}-1 / v}{\left(s_{v}\right)^{2}}=0 \text {. }
$$

Thus (17) is true for a special sequence $\left\{s_{v}\right\}$. To show (17) for all se quences $\left\{s_{v}\right\}$ tending to zero, as $v \rightarrow \infty$, we distinguish two cases:

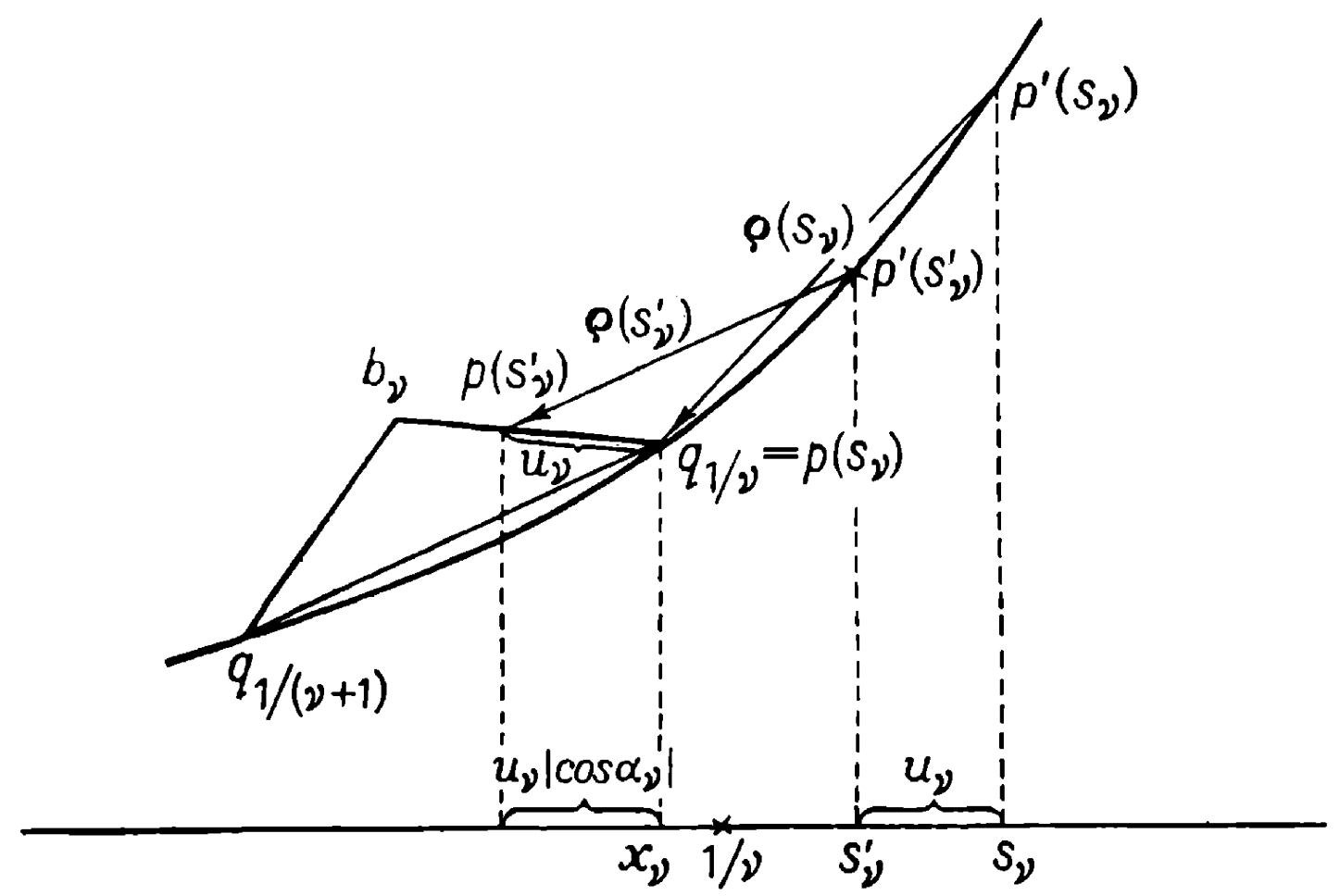

Fig. 1

$1^{\circ}$ Let $\tau_{v}$ for all $v$ lie on $E^{2}$ and let $\left\{s_{v}^{\prime}\right\}$ be such a sequence that $s_{v} \leqslant s_{v}^{\prime} \leqslant s_{y}+\delta_{v}$, where $s_{v}=\left(\nu^{2}+1\right) / \nu^{3}$. Denote by $u_{v}$ the length of the segment $q_{1 / v} p\left(s_{v}^{\prime}\right)$. The abscissas of $p^{\prime}\left(s_{v}^{\prime}\right)$ and $p\left(s_{v}^{\prime}\right)$ are equal to $s_{v}^{\prime}=s_{v}-u_{v}$ and $x_{v}-u_{v}\left|\cos a_{v}\right|$, respectively, where $a_{v}$ is the measure of the angle which yields $\overrightarrow{q_{1 / v} b}$, with $e_{1}$. Further, we have

$$
\lim _{x \rightarrow \infty} \frac{\delta_{v}}{\left(s_{v}\right)^{2}}=\frac{1}{2}
$$

Hence in view of $0 \leqslant u_{v} \leqslant \delta$, we obtain

$$
\lim _{v \rightarrow \infty} \frac{u_{v}}{\left(s_{v}-u_{v}\right)^{2}}=\lim _{v \rightarrow \infty} \frac{u_{v} /\left(s_{v}\right)^{2}}{\left(1-u_{v} / s_{v}\right)^{2}}=a_{v} \leqslant \frac{1}{2}, \quad a_{v}>0,
$$

and

$$
\lim _{r \rightarrow \infty} \frac{u_{p}}{s,}=0
$$


Thus

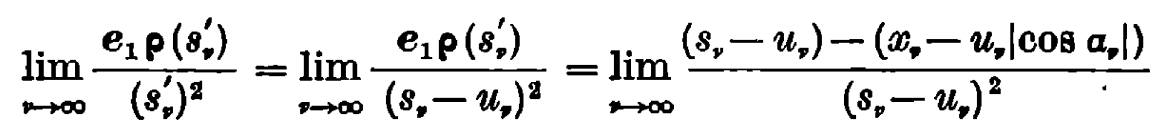

$$
\begin{aligned}
& =\lim _{\nu \rightarrow \infty} \frac{\left(s_{v}-x_{v}\right)+u_{v}\left(1-\left|\cos \alpha_{v}\right|\right)}{\left(s_{\nu}-u_{\nu}\right)^{2}}=0 .
\end{aligned}
$$

In fact, by (19) and because of $a_{\nu} \rightarrow 0, \lim _{r \rightarrow \infty} \frac{u_{\nu}\left(1-\left|\cos a_{\nu}\right|\right)}{\left(s_{\nu}-u_{v}\right)^{2}}=0$. By (17), (20) and in virtue of the equivalence of sequences $\left\{x_{v}\right\}$ and $\{1 / \nu\}$, $\lim _{v \rightarrow \infty} \frac{s_{p}-x_{v}}{\left(s_{\nu}-u_{v}\right)^{2}}=0$.

$2^{\circ}$ If $s_{v+1}-\delta_{v} \leqslant s^{\prime} \leqslant s_{v+1}$, i. e. if $p\left(s_{v}^{\prime}\right) \in b_{v} q_{1 /(v+1)}$, we proceed as in $1^{\circ}$ and we obtain $\lim _{v \rightarrow \infty} \frac{e_{1} p\left(s_{p}^{\prime}\right)}{\left(s_{p}^{\prime}\right)^{2}}=0$ also in this case.

For any $s,\left|e_{1} p(s)\right|$ takes its greatest value when $\tau_{v}$ lies on $E^{2}$. Hence or every position of $\tau_{v}$ we have

$$
\lim _{s \rightarrow 0} \frac{e_{1} \rho(s)}{s^{2}}=0
$$

The absolute values of the second and third coordinates of $p(s)$ are smaller than the absolute value of the first, a least starting from an 8 . Therefore

$$
\lim _{s \rightarrow \infty} \frac{e_{2} p(s)}{s^{2}}=\lim _{s \rightarrow \infty} \frac{e_{3} p(s)}{s^{2}}=0
$$

Thus we have shown that (17) is true.

Now define the position of $\tau$, and the sequence $\left\{s_{v}^{1}, s_{v}^{2}, s_{v}^{3}\right\}$ in the following way. Let $\tau_{v}$, for $v$ even, be in any admissible position (see (ii)). Take two points $p\left(s_{v}^{1}\right), p\left(s_{v}^{2}\right)$ on $q_{1 / v}, b_{v}, b_{v} q_{1 /(v+1)}$ sufficientily near to $q_{1 / v}$ and $q_{1 /(v+1)}$ respectively, i. e. so near that in some position of $\tau_{v+1}$ the line $p\left(s_{v}^{1}\right) p\left(s_{v}^{2}\right)$ intersects $q_{1 /(v+1)} b_{v+1}$ at a point $p\left(s_{v}^{\prime 3}\right)$. Then move $s_{v}^{\prime 3}$ into a new position $s_{v}^{3}$ near enough to $s_{v}^{\prime 3}$ in such a way that the circle $S_{v}^{1}$ through $p\left(s_{v}^{1}\right), p\left(s_{v}^{2}\right), p\left(s_{v}^{3}\right)$ has a radius greater than $2+\varepsilon, \varepsilon>0$. Such a displacement of $s_{v}^{\prime 3}$ is possible for every $v$. Thus the limit circle of the sequences $\left\{\mathcal{S}_{*}^{1}\right\}$, as $\nu \rightarrow \infty$, does not exist, or, if it exists, it is different from 2, i. e. from the radius of the osculating circle $S_{3}^{1}$. It is clear that the sequence $\left\{P_{v}\right\}=\left\{p\left(s_{v}^{1}\right), p\left(s_{v}^{2}\right), p\left(s_{v}^{3}\right)\right\}$, where $v$ is even, is not normal, since we have $\lim _{n \rightarrow \infty} 8_{v}^{1} / \delta_{v}^{2}=1$. 
To make our Example more instructive we can take a normal sequence $\left\{P_{v^{\prime}}^{(1)}\right\}$, for $\nu^{\prime}$ odd, and form a new sequence $\left\{P_{\nu^{\prime \prime}}^{(2)}\right\}, v^{\prime \prime}=1,2,3, \ldots$, which contains $\left\{\boldsymbol{P}_{v^{\prime}}^{(1)}\right\}$ and the $\left\{\boldsymbol{P}_{v}\right\}$ just defined as its subsequences. $\left\{\boldsymbol{P}_{v^{\prime \prime}}^{(2)}\right\}$ does not yield, of course, any osculating sphere.

I am greatly indebted to Professor $\mathrm{S}$. Gołąb for the careful reading of the manuscript of this paper and for the remarks which enabled to improve the text.

\section{References}

[1] A. Denjoy, Sur l'intégration des coefficientes différentiels d'ordre supérieur, Fund. Math. 25 (1935), p. 273-326.

[2] S. Drobot, On the foundation of Dimensional Analysis, Studia Math. 14 (1953), p. 84-99.

[3] S. Fudali, k-wymiarowa hipersfera ścisle styczna do krzywej w n-wymiarowej przestrzeni euklidesowej ( $k$-dimensional osculating hypersphere to ourve in $n$-dimensional Euclidean space), doctor thesis, not published.

[4] V. Jarnik, $O$ kružnici kř́ivosti, Casopis pro péstováni matem. a fisiky, roč. 73 (1949), D 37 - D 51.

[5] T. Rachwal, L'ordre du contact d'une courbe régulière avec la sphère osculatrice, Ann. Pol. Math. 6 (1958), p. 33-43.

[6] - Oontaot d'une courbe régulière aveo la sphère osculatrice dans un point d'inflexion $R_{3}$, ibidem 10 (1961), p. $35-39$.

[7] - Oontàot d'une courbe régulière avec les hypersphères tangentes en un point d'inflexion d'ordre $n-1$ ('espace euclidean d $n$ dimension), Czechosłovak Math. J. 11 (86), (1961), p. 570-586.

[8] H. A. Schwarz, Verallgemeinerung eines analytischen Fundamentalsatzes, Annali di Math. Pura ed Applicata, T. X (1880-82), p. 129-136. 